LBL-37525

UC-404

\title{
Surface Recombination in Semiconductors
}

\author{
J.M. Langer* and W. Walukiewicz** \\ *Institute of Physics \\ Polish Academy of Sciences \\ Al. Lotnikow 32/46 \\ 02668 Warsaw, Poland \\ **Center for Advanced Materials \\ Materials Sciences Division \\ Lawrence Berkeley National Laboratory \\ University of California \\ Berkeley, California 94720
}

July 1995

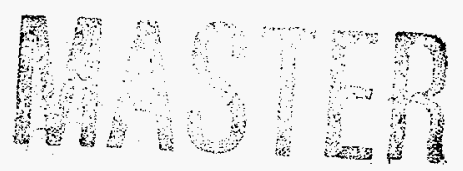

This work was supported by the Director, Office of Energy Research, Office of Basic Energy Sciences,

Materials Sciences Division, of the U.S. Department of Energy under Contract No. DE-AC03-76SF00098. $\because$ 


\section{DISCLAIMER}

This report was prepared as an account of work sponsored by an agency of the United States Government. Neither the United States Government nor any agency thereof, nor any of their employees, make any warranty, express or implied, or assumes any legal liability or responsibility for the accuracy, completeness, or usefulness of any information, apparatus, product, or process disclosed, or represents that its use would not infringe privately owned rights. Reference herein to any specific commercial product, process, or service by trade name, trademark, manufacturer, or otherwise does not necessarily constitute or imply its endorsement, recommendation, or favoring by the United States Government or any agency thereof. The views and opinions of authors expressed herein do not necessarily state or reflect those of the United States Government or any agency thereof. 


\section{DISCLAIMER}

\section{Portions of this document may be illegible in electronic image products. Images are produced from the best available original document.}




\title{
SURFACE RECOMBINATION IN SEMICONDUCTORS
}

\author{
J. M. LANGER ${ }^{1}$ AND W. WALUKIEWICZ ${ }^{2}$ \\ ${ }^{1}$ Institute of Physics Polish Academy of Sciences, Al. Lotnikow 32/46, 02668 Warsaw, Poland \\ ${ }^{2}$ Center for Advanced Materials, Materials Sciences Division, Lawrence Berkeley National \\ Laboratory, Berkeley, CA 94720, USA
}

Keywords: nonradiative recombination, minority carrier lifetime, defects

\begin{abstract}
We propose two general criteria for a surface defect state to act as an efficient, nonradiative recombination center. The first is that the thermal ionization energy should not deviate from the mid-gap energy by more than the relaxation energy of the defect. In this case the activation energy for the recombination is given by the barrier for the capture of the first carrier, whereas the second carrier is captured athermally. The second citerion is related to the position of the average dangling bond energy relative to the band edges. If, as in the cases of InP or InAs, it is located close to a band edge, a low surface recombination velocity is expected. However a much faster recombination is predicated and experimentally observed in the materials with the average dangling bond energy located close to the mid-gap. The relevance of these criteria for the novel wide-gap optoelectronic materials is discussed.
\end{abstract}

\section{Introduction}

Minority carrier lifetime is a basic parameter determining the performance of a large variety of semiconductor devices. Over the last few decades a considerable effort was aimed at understanding and controlling of the recombination processes in semiconductor materials. It is now widely recognized that highly localized deep defects in the bulk and on semiconductor surfaces are extremely efficient recombination centers [1]. Thus it has been found that a recombination via deep levels associated with transition metal impurities can be a dominant mechanism controlling the minority carrier lifetime in Si [1]. Also, the native defects in the bulk and on semiconductor surfaces can provide channels for the recombination of the minority carriers. The issue of recombination via imperfections at semiconductor surfaces and at internal interfaces is becoming especially critical for the novel technologies with steadily decreasing size of semiconductor devices [2]. Here we show how our present understanding of the general properties of native defects can be used to evaluate the trends in the surface recombination velocities in different compound semiconductors.

In general the lifetime of minority carriers is given by the equation:

$$
\frac{1}{\tau_{\text {minority }}}=\frac{1}{\tau_{\text {radiative }}}+\frac{1}{\tau_{\text {defects }}}+\frac{1}{\tau_{\text {surface }}}
$$

It is customary to relate nonradiative surface recombination to the surface recombination velocity s and the sample thickness $d$. For the sample with two surfaces (or interfaces) $a$ and $b$

$$
\frac{1}{\tau_{\text {surface }}}=\frac{s_{a}+s_{b}}{d}
$$

In devices, for which the active thickness is small (like quantum wells or even thin film light emitters) surface recombination is the ultimate factor limiting the quantum efficiency $\eta$ of the radiative recombination,

$$
\eta=\frac{\tau_{\text {minority }}}{\tau_{\text {radiative }}} \approx \frac{d}{2 s \tau_{\text {radiative }}}
$$


From the above considerations it becomes clear that semiconductors with inherently large surface recombination velocity $\mathbf{s}$ are not useful as light emitters irrespective of other material parameters [3].

There are two ways to lower the surface recombination rate. One is a chemical passivation of the surface[3], while the others involves the formation of a potential barrier that would prevent minority carriers from reaching the surface. Such a potential can be accomplished by making an appropriate heterojunction with low interface recombination rate (e.g. AlGaAs on GaAs) [4] or by strong doping of the surface region that electrostatically bends the bands in a way that would confine minority carriers in the bulk [5].

A very large spread exists in the values of the intrinsic surface recombination velocities among semiconductors. Good examples are GaAs (fast) and InP (slow) or a similar pair namely Si and Ge. A understanding of this puzzle could have significant practical implications for minority carrier devices, as well as for basic physics. The experience gathered over the years from surface studies indicates that despite the fact that it must be real defect levels at the surface through which the nonradiative recombination takes place, it is the host band structure that ultimately controls the position of these levels. Quite similar problems were encountered in the discussion of the mechanism of Schottky barrier formation [6-9], heterojunction band offsets $[6,7,10]$, Fermi level stabilization of heavily irradiated semiconductors $[8,11]$ and many other phenomena in the physics of semiconductors. It is tempting to assume that all these observations must have a common explanation and that the chemical trends are transferable from one phenomenon to another. Such a notion led us to the formulation of two basic criteria which determine which of the surface defects can act as a fast recombination centers and also to predict the chemical trends in the surface recombination rates among various semiconductors.

\section{Mid-gap states as efficient recombination centers: First criterion}

The recombination statistics for the surface states is the same as for bulk defects. Therefore by substituting in the Shockley-Hall-Read statistics the bulk defect parameters by effective surface defect parameters (densities $\mathrm{N}_{\mathrm{s}}$, trapping cross sections $\mathrm{s}_{\mathrm{s}}$ and surface Fermi level), it is immediately clear that the surface recombination has a maximum when the surface Fermi level is at the mid-gap and coincides with the energy of the surface traps $[3,12,13]$. The recombination velocity is proportional to the density of surface states and is given by the smaller of the two capture cross sections for holes and electrons. However, an order of magnitude estimate based on an analysis of the recombination statistics shows that for GaAs the cross section for trapping has an unreasonably low value of less than $10^{-20} \mathrm{~cm}^{2}[3,12]$. The simplest way to understand this result is to assume [12] that the capture is a thermally activated process and is given by a standard multiphonon capture formula where $\mathrm{E}_{\mathrm{B}}$ is the energy barrier for the carrier capture.

$$
\sigma=\sigma_{0} \exp \left(-\mathrm{E}_{\mathrm{B}} / \mathrm{k} \tau\right)
$$

Eq. (4) has been obtained assuming that the defects responsible for the recombination are localized and strongly coupled to the lattice. According to all recent theoretical and experimental works these assumptions are well justified for native defects at semiconductor surfaces. Although the energies of these defects may not be necessary the same as those of the bulk defects, they retain most of the characteristic features, especially large lattice relaxation, of the analogous bulk defects $[12,14,15]$.

Let us now consider how carriers recombine via a deep defect strongly coupled to the lattice. A simple, but incorrect notion, is that the effective barrier height for capture is the sum of the capture barriers for electrons and holes. Suppose that the defect is initially empty, hence the first carrier captured is an electron. As is shown in Fig. 1 after the capture over the barrier $E_{B}$ the vibronic coordinate changes from $Q_{1}$ to $Q_{B}$. Remembering that for a quantum oscillator the maximum probability is the same for both turning points, i.e., on both sides of the CC parabola, a capture of a hole at $Q_{B}$ ' occurs. before the filled defect reaches its equilibrium at $Q_{2}$. Therefore, if only the energy of the crossing point at $Q_{B}$ is below the energy of the electron captured at $Q_{B}$, the hole is captured athermally without any barrier. The same argument applies when the hole is being captured first. 
A simple consideration of the parameters of the CC diagram shown in Fig. 1 leads to the following criterion for a defect to be an effective recombination center,

$$
\mathrm{E}_{\mathrm{g} / 2}-\mathrm{E}_{\text {relax }}<\mathrm{E}_{\text {trap }}<\mathrm{E}_{\mathrm{g} / 2}+\mathrm{E}_{\text {relax }}
$$

where $E_{g}$ is the energy gap, $E_{\text {trap }}$ is the thermal ionization energy and $E_{\text {relax }}$ is the relaxation energy which is the difference between optical and thermal ionization energies of the defect.

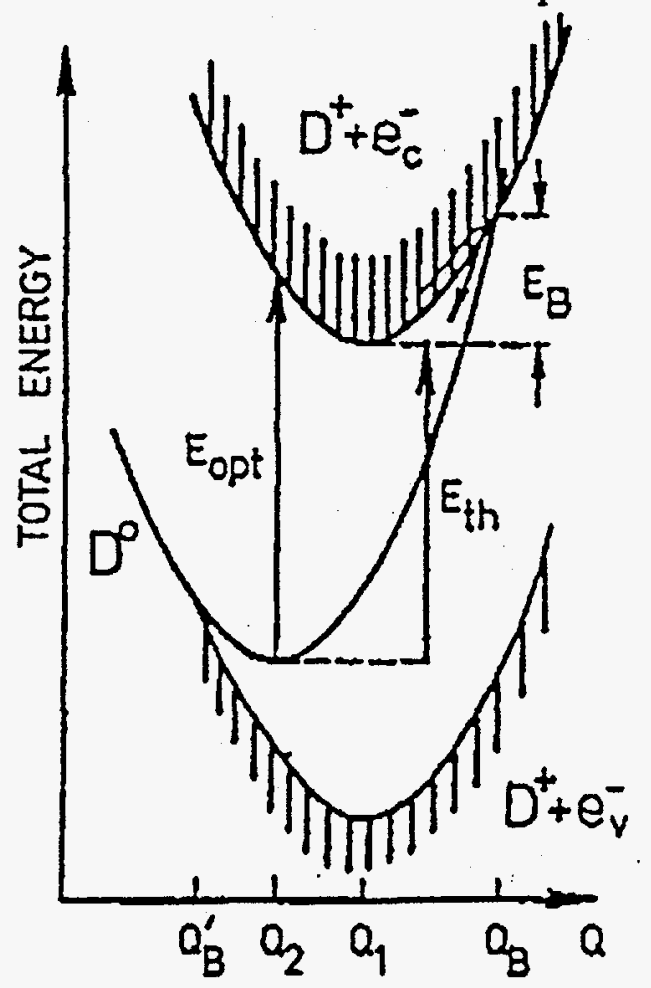

Fig. 1 Schematic configurational coordinate $(\mathrm{CC})$ diagram for a highly localized defect strongly coupled to the semiconductor crystal lattice.
The relaxation energy, $E_{\text {relax }}$ represents a gain in the total energy of the vibrating defect moving from the equilibrium position $Q_{1}$ to the position $Q_{2}$. For most deep native defects this energy is a large fraction of an electronvolt and it is increasing with the ionicity of the material. Consequently, if the levels of the surface defects are close to middle of the gap and are strongly coupled to the lattice they are likely to be fast recombination centers. In such a case a trapping barrier occurs only for the first carrier (electron or hole) whereas the capture of the second carrier (hole or electron) is athermal. The position of the Fermi level at the surface dictates which carrier will be captured first.

This simple quantum analysis of the capture process provides not only an intuitive argument about the fastest recombination through mid-gap levels, but also offers a microscopic explanation of the role of local vibrations in the recombination process. Similar arguments lead Bartram and Stoneham to formulate a general criterion of the intra-defect luminescence in defects strongly coupled to the lattice in ionic crystals [16]. Finally, it is worth pointing out that the discrepancies between spectroscopic measurements of the surface states distribution [17] and most of electrical measurements have obvious explanations in terms of the defect-lattice coupling for which the thermal ionization energy is always smaller than the optical ionization energy.

\section{Surface states and the dangling bonds: Second criterion}

The first criterion relates the location of the defect energy level to its properties as a recombination center. Unfortunately, in most cases the identity and thus also the positions of the energy levels associated with a specific recombination center are not known making it difficult to deduce the surface recombination velocities for different materials. To address this issue we devise the second criterion which utilizes recently discovered general properties of the highly localized native defects in semiconductors.

The criterion originates from the observation that the location of a charge transition state associated with a broken bonds on the surface is uniquely determined by a universal energy reference known as a neutrality level or Fermi level stabilization energy $[6-9,12]$ This energy reference has been used to predict the heterojunction band offsets $[6,7,10]$, Schottky barrier heights $[6,8,12]$ and the Fermi level stabilization in heavily damaged semiconductors [8, 11]. The position of the Fermi level stabilization energy, $E_{F S}$ for different III-V semiconductors is shown in Fig. 2.

Assuming that the defect states responsible for the surface recombination are of a broken bond character, we can use the location of $\mathrm{E}_{\mathrm{FS}}$ or the average dangling bond energy relative to the band edges to qualitatively predict the chemical trends in the surface recombination velocity.

According to the first criterion a slow surface recombination is expected in semiconductors with $E_{F S}$ close to the band edges. However if it is located near midgap, then a much faster surface recombination is predicted. This procedure can use the $E_{F S}$ computed as a neutrality level $[6,10,12$, 
15] determined from the position of the Fermi level in heavily irradiated semiconductors [8-11] or from the Schottky barrier heights $[6-8,12,18,19]$.

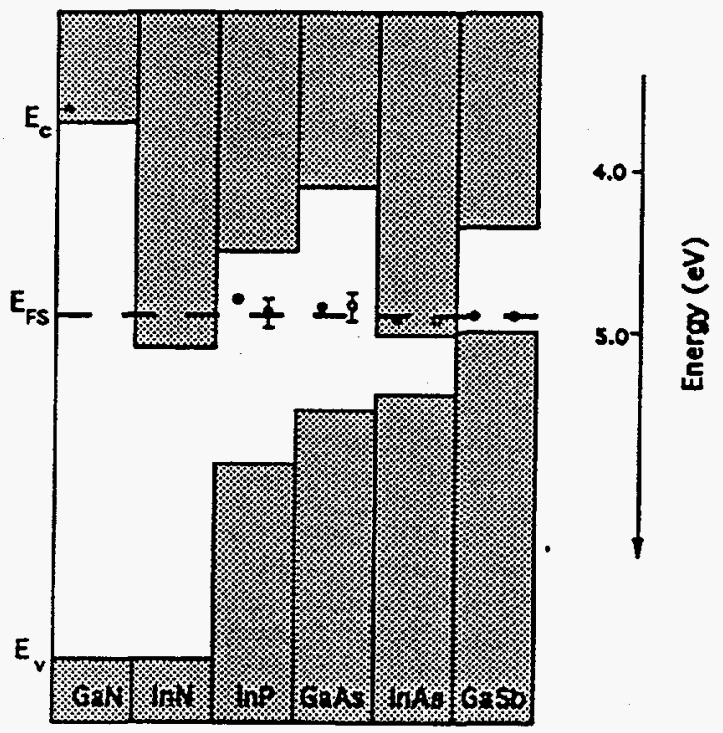

Fig. 2 Position of the Fermi level stabilization energy in different III-V semiconductors determined from the Schottky barrier heights ( 0 ) and from the position of the Fermi level in materials heavily damaged with high energy particles $(\bullet)$.

Since the recombination processes are of critical importance for optoelectronic devices it is worth inspecting how the outlined concepts apply to the wide gap materials that could be used for short wavelength light emitters. As is shown in Fig. 2 in GaN and especially in InN EFs is predicted to be located in the upper half of the band gap. This indicates that the localized, dangling bond defects on surfaces or at internal interfaces are not very efficient recombination centers in these materials. Indeed, it has been reported recently that very efficient light emitting diodes were made with GaN containing extremely high densities of extended defects [21].

Among II-VI compounds the average dangling bond energy is very close to the conduction band edges in CdS and CdSe. Both materials are very efficient light emitters. They are widely used as nanocrystals with large quantum efficiencies [22]. On the other hand CdTe has its EFs close the midgap energy and consequently fast surface recombination velocities are expected in this material.

Despite a success in predicting the trends in the surface recombination velocities in various materials a caution has to be exercised when applying this concept to very wide-gap, ionic semiconductors. In such materials there is a large ionicity difference between the two component elements and thus also a large difference in the formation energies of the defects located on two different sublattices and the concept of the common energy reference is not uniquely defined.

\section{Conclusions}

We propose a simple and intuitive explanation of the large differences in the surface recombination velocities observed in different semiconductors. The explanation stems from a recent observation of the correlation of the bulk and interface properties of semiconductors with the position of the average dangling bond or Fermi level stabilization energy in semiconductors. It becomes more evident now that most native defects that govern the properties of an untreated surface are localized and strongly coupled to the lattice. Such defect may be strong nonradiative recombination channels 
if they lie within the energy region centered at middle of the gap within relaxation energy of the defect undergoing ionization neutralization.

\section{Acknowledgments}

Discussions with E:E. Haller are gratefully acknowledged. The part of the work performed at LBNL was supported by the Director, Office of Energy Research, Office of Basic Energy Science, Materials Sciences Division of the U.S. Department of Energy under contract No. DE-ACO376 SF00098.

\section{References}

[1] A.R. Peaker and B. Hamilton, Chemtronics 3, 194 (1988)

[2] J.M. Langer, Europhysics News, Bull. European Phys. Soc.18, 142(1987)

[3] D.E. Aspnes, Surface Science 132, 406 (1983)

[4] R.J. Nelson and R.G. Sobers, J. Appl. Phys. 41, 6103 (1978)

[5] J.S. Rimmer, J.M. Langer, M. Missous, J.H. Evans, I. Poole, A.R. Peaker and K. Singer, Materials Science and Engineering B9, 375 (1991)

[6] J. Tersoff, Phys. Rev. Lett. 52, 465 (1984), Phys. Rev. B30, 4874 (1984)

[7] F. Flores and C. Tejedor, J. Phys. C20, 145 (1987)

[8] W. Walukiewicz, J. Vac. Sci. Technology, B6, 1257 (1988); Appl. Phys. Lett. 54, 2094 (1989)

[9] H. Hasegawa and H. Ohno, J. Vac. Sci. Technology, B4, 1130 (1986)

[10] J.M. Langer and H. Heinrich, Phys. Rev. Lett. 55, 1414 (1985), J.M. Langer, C. Delerue, M. Lannoo and H. Heinrich, Phys. Rev. B38, 7723 (1988),

[11] W. Walukiewicz, Materials Science Forum. 143-147, 519 (1994)

[12] M. Lannoo, Revue Phys. Appl. 22, 789 (1987)

[13] D.D. Nolte, Solid-State Electronics 33, 295 (1990)

[14] J.M. Moison, M Van Rompay and M. Bensoussan, Appl. Phys. Lett. 48, 1362 (1986)

[15] M. Lannoo and P. Friedel: Atomic and Electronc Structure of Surfaces, Theoretical Foundations (Springer-Verlag, Berlin, 1991).

[16] R.H. Bartram an A.M. Stoneham, Sol. State Commun. 17, 1593 (1975)

[17] G. Lengel, R. Wilkins, G. Brown, M. Weimer, J. Gryko and R. Allen, Phys. Rev. Lett. 72, 836 (1994)

[18] J. M. Langer and P. Revva, Materials Science Forum 83-87, 1545 (1992), P. Revva, J. M. Langer, M. Missous and A. R. Peaker: J. Appl. Phys. (1993)

[19] I. Lindau and T. Kendelewicz, CRC Crit. Rev. Sol. State Mat. Sci. 13, 27 (1986)

[20] H.C. Casey Jr. and E. Buehler, Appl. Phys. Lett. 30, 247 (1977)

[21] S. D. Lester, F. A. Ponce, M. G. Craford and D. A. Steigerwald, Appl. Phys. Lett. 66, 1249, (1994)

[22] see e.g. L. E. Brus, Appl. Phys. A53, 465 (1991) 